\title{
Clinical outcomes of various continued antiplatelet therapies in patients who were administered DAPT following the implantation of drug-eluting stents and developed gastrointestinal hemorrhage
}

\author{
YUJIE GUO $^{1,2}$ and JINRU WEI ${ }^{3}$ \\ ${ }^{1}$ Department of Cardiology, First Affiliated Hospital of Guangxi Medical University, Guangxi Cardiovascular Institute, \\ Nanning, Guangxi 530021; ${ }^{2}$ Department of Cardiology, Liuzhou General Hospital, Liuzhou, Guangxi 545006; \\ ${ }^{3}$ Department of Cardiovascular Disease, The First People's Hospital of Nanning City, \\ Nanning, Guangxi 530022, P.R. China
}

Received April 3, 2015; Accepted May 3, 2016

DOI: $10.3892 /$ etm.2016.3378

\begin{abstract}
Although an increasing number of patients accept dual antiplatelet therapy (DAPT) following implantation of drug-eluting stents (DES) for coronary heart disease (CHD), the proportion of patients with DAPT who subsequently develop gastrointestinal hemorrhage continues to increase. To ensure the clinical outcomes from DES, it is important to formulate a novel continued antiplatelet therapy for patients who were administered DAPT and subsequently develop gastrointestinal hemorrhage following DES implantation. The present study aimed to evaluate the effects of continued aspirin, clopidogrel or DAPT use on the incidence of clinical adverse events and gastrointestinal rebleeding in patients who received DAPT and subsequently developed gastrointestinal hemorrhage following implantation of DES for CHD. Between 2004 and 2010, 108 consecutive patients receiving DAPT developed gastrointestinal hemorrhage following DES implantation for CHD at Liuzhou General Hospital (Liuzhou, Guangxi). These patients were divided into three groups according to the novel antiplatelet therapy. The occurrence of major adverse cardiac events (MACE), including cardiac death, non-fatal myocardial infarction, heart failure or target vessel revascularization, net adverse clinical events (NACE), including major bleeding, stroke or MACE, and gastrointestinal rebleeding during clinical follow-up following the initial procedure were compared among these three groups. The results of this analysis demonstrated that the occurrence rate of MACE, NECE and gastrointestinal rebleeding was not significantly different among these groups $(\mathrm{P}>0.05)$. Furthermore, survival
\end{abstract}

Correspondence to: Dr Jinru Wei, Department of Cardiovascular Disease, The First People's Hospital of Nanning City, 89 Qixing Road, Nanning, Guangxi 530022, P.R. China E-mail: jinruweidj@aliyun.com

Key words: dual antiplatelet therapy, gastrointestinal hemorrhage, major adverse cardiac events, net adverse clinical events analysis was performed and although the survival curves of MACE and NECE were not significantly different among these groups, gastrointestinal rebleeding was demonstrated to be significantly different among the three groups $(\mathrm{P}<0.05)$, and continued aspirin or clopidogrel use was superior to continued DAPT. In conclusion, the results of the present study indicated that there were no significant differences in the clinical effectiveness and safety of continuing antiplatelet monotherapy or DAPT in patients who are administered DAPT and experience gastrointestinal hemorrhage following DES implantation. As for the prevention of recurrent bleeding, antiplatelet monotherapy was demonstrated to be superior to DAPT. Moreover, the treatment of patients who are administered DAPT and experience gastrointestinal hemorrhage following DES implantation must involve an evaluation of the risk of complications, including stent thrombosis, continuous bleeding and recurrent hemorrhage.

\section{Introduction}

Coronary artery disease (CAD) is the most prevalent cause of death in patients suffering from cardiovascular diseases (1). It is well-established that the complications of CAD include acute coronary syndrome (ACS), arrhythmias, heart failure and sudden death. ACS, particularly acute myocardial infarction (AMI), is a major public health concern, as it is the cause of $\sim 75 \%$ of all CHD deaths and accounts for $\sim 50 \%$ of all CHD hospital admissions (2). Percutaneous coronary intervention (PCI) and thrombolytic therapy are currently used in clinics as the predominant methods of treating AMI. Dual antiplatelet therapy (DAPT) is recommended for the treatment of coronary heart disease, which includes aspirin and an ADP receptor blocker (3). Regardless of which therapy is used, patients are recommended to accept anticoagulant or antiplatelet therapies, including aspirin, clopidogrel and warfarin, in order to prevent the onset of recurrent infarction following intervention (4). Bleeding, including intracranial hemorrhage and gastrointestinal hemorrhage, remains the predominant adverse effect of antiplatelet therapy (5). However, physicians cannot terminate antiplatelet therapy due to potential high incidence of recurrent 
Table I. Clinical events observed indicators.

\begin{tabular}{lccccr}
\hline Variable & Group A & Group B & Group C & $\chi^{2}$ value & P-value \\
\hline Patients & 18 & 60 & 25 & & \\
MACE, n (\%) & $1(5.6 \%)$ & $3(5.0 \%)$ & $1(4.0 \%)$ & 0.061 & 0.972 \\
NACE, n (\%) & $1(5.6 \%)$ & $4(6.7 \%)$ & $2(8.0 \%)$ & 0.103 & 0.955 \\
RH, n $(\%)$ & $2(11.1 \%)$ & $5(10.0 \%)$ & $4(16.0 \%)$ & 1.092 & 0.582 \\
\hline
\end{tabular}

MACE, major adverse cardiovascular events; NACE, net adverse cardiovascular events; RH, recurrent hemorrhage.

Table II. Survival analysis of clinical events.

\begin{tabular}{|c|c|c|c|c|c|c|}
\hline \multirow[b]{2}{*}{ Method } & \multicolumn{2}{|c|}{ MACE } & \multicolumn{2}{|c|}{ NACE } & \multicolumn{2}{|c|}{ Recurrent hemorrhage } \\
\hline & $\chi^{2}$ value & P-value & $\chi^{2}$ value & P-value & $\chi^{2}$ value & P-value \\
\hline Long-rank & 2.929 & 0.231 & 3.697 & 0.157 & 5.336 & 0.069 \\
\hline Breslow & 1.444 & 0.486 & 2.763 & 0.251 & 6.909 & 0.032 \\
\hline Tarone-Ware & 1.971 & 0.373 & 3.142 & 0.208 & 6.419 & 0.040 \\
\hline
\end{tabular}

Significant differences $(\mathrm{P}<0.05)$ are indicated in bold. MACE, major adverse cardiovascular events; NACE, net adverse cardiovascular events

infarction. Therefore, the present study investigated patients admitted to Liuzhou General Hospital (Liuzhou, Guangxi) between 2004 and 2010, who were suffering from gastrointestinal hemorrhage following DAPT after the implantation of drug eluting stents (DES) in order to compare the efficacy of various types of continued antiplatelet therapy. The results of the present study may help elucidate the most effective method of continued antiplatelet therapy for patients who were treated with DAPT following DES implantation and subsequently developed gastrointestinal hemorrhage.

\section{Materials and methods}

Study participants. Patients were eligible for the present study if they had experienced recurrent hemorrhage within one year of DES implantation and DAPT, and had no relevant co-morbidities. A total of 108 patients (aged 38-78 years) were recruited from our hospital between 2004 and 2010. Exclusion criteria included i) The patient died during PCI or within $24 \mathrm{~h}$ of the surgery; and ii) the patient exhibited bleeding diathesis or was allergic to antiplatelet drugs. The present study was approved by the Institutional Ethics Committee, and written informed consent was obtained from all study participants.

Grouping. Patients were randomly assigned to three groups: Group A, $100 \mathrm{mg}$ aspirin + proton pump inhibitor (PPI; n=18); group B, 75 mg clopidogrel + PPI (n=60); and group C, DAPT + PPI $(n=25)$. Treatment was administered for 14 (2-25) months. PPI treatment included esomeprazole (20 mg q.d.), rabeprazole (20 mg q.d.), or pantoprazole (40 mg q.d.). Baseline characteristics, including age, ethnicity, basic treatment, condition of PCI surgery, were recorded for all three groups and no significant heterogeneity was detected. Therapies administered post-PCI surgery were identical in each group.
Follow up. All patients were contacted by telephone or attended an out-patient follow up. During follow-up, the patient's state of health and major evaluation criteria were recorded, including: Major adverse cardiovascular events (MACE), which included heart failure, AMI and cardiac death; net adverse clinical events (NACE), which included bleeding stroke and MACE; and recurrent of gastrointestinal hemorrhage. The dates of these incidents were recorded.

Statistical analysis. Student's t-test or analysis of variance was performed to compare continuous variables between populations. Two-tailed Fisher's exact test or $\chi^{2}$ test was used to compare categorical variables. Survival analysis was used to compare patients' survival data and was performed using the Log-rank, Breslow and Tarone-Ware methods (6-8). Data were presented as the mean \pm standard deviation. $\mathrm{P}<0.05$ was considered to indicate a statistically significant difference. Statistical analyses were performed using SPSS 15.0 software (SPSS, Inc., Chicago, IL, USA).

\section{Results}

DAPT and monotherapy induce similar incidence rates of MACE, NACE and recurrent hemorrhage. The mean follow-up period for the present study was 14 months (range 2-25 months). Five patients were lost to follow-up (changed telephone number or stopped attending out-patient follow-up), resulting in a dropout rate of $4.6 \%$; therefore 103 patients were postoperatively followed-up in the three groups: A $(n=18), B(n=60)$ and C $(n=25$; Table I). The incidence rates of MACE in groups $A(n=1), B(n=3)$ and $C(n=1)$ were 5.6, 5.0 and $4.0 \%$, respectively $(\mathrm{P}=0.97)$. Incidence rates of NACE in groups $A(n=1), B(n=4)$ and $C(n=2)$ were 5.6, 6.7 and $8.0 \%$, respectively $(\mathrm{P}=0.95)$ and the recurrent hemorrhage 
Table III. Survival analysis of recurrent hemorrhage.

\begin{tabular}{|c|c|c|c|c|c|c|}
\hline \multirow[b]{2}{*}{ Variable } & \multicolumn{2}{|c|}{ Group A } & \multicolumn{2}{|c|}{ Group B } & \multicolumn{2}{|c|}{ Group C } \\
\hline & $\chi^{2}$ value & P-value & $\chi^{2}$ value & P-value & $\chi^{2}$ value & P-value \\
\hline \multicolumn{7}{|c|}{ Long-rank method } \\
\hline Group A & & & 0.205 & 0.651 & 4.054 & 0.044 \\
\hline Group B & 0.205 & 0.651 & & & 2.897 & 0.089 \\
\hline Group C & 4.054 & 0.044 & 2.897 & 0.089 & & \\
\hline \multicolumn{7}{|c|}{ Breslow method } \\
\hline Group A & & & 1.215 & 0.270 & 3.882 & 0.049 \\
\hline Group B & 1.215 & 0.270 & & & 3.959 & 0.047 \\
\hline Group C & 3.882 & 0.049 & 3.959 & 0.047 & & \\
\hline \multicolumn{7}{|c|}{ Tarone-Ware method } \\
\hline Group A & & & & & & \\
\hline Group B & 0.796 & 0.372 & 0.796 & 0.372 & 3.987 & 0.046 \\
\hline Group C & 3.987 & 0.046 & 3.605 & 0.058 & 3.605 & 0.058 \\
\hline
\end{tabular}

Significant differences $(\mathrm{P}<0.05)$ are indicated in bold.

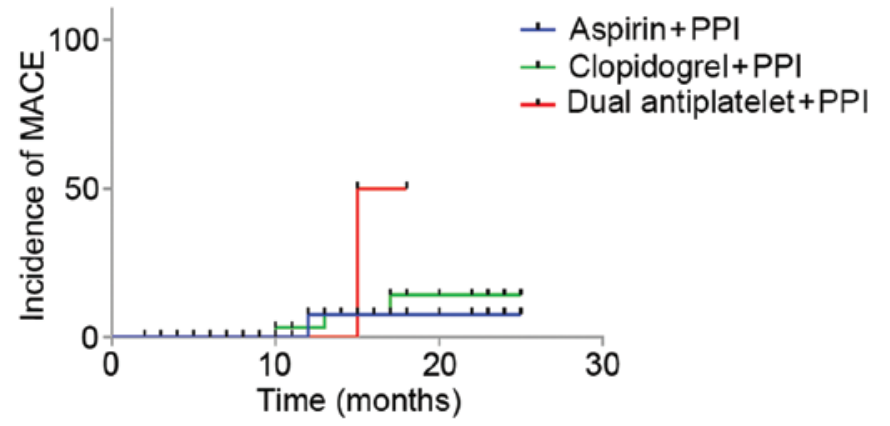

Figure 1. Incidence of major adverse cardiovascular events among the three groups.

rates in groups $\mathrm{A}(\mathrm{n}=2), \mathrm{B}(\mathrm{n}=5)$ and $\mathrm{C}(\mathrm{n}=4)$ were $11.0,10.0$ and $16.0 \%$, respectively $(\mathrm{P}=0.58)$. No significant differences in the incidence rates of MACE, NACE and recurrent hemorrhage were detected among the three groups $(\mathrm{P}>0.05)$.

DAPT increases gastrointestinal rebleeding rates. Survival analysis was performed using the Log-rank, Breslow and Tarone-Ware methods. As demonstrated in Table II, survival analysis of MACE and NACE outcomes demonstrated that there were no significant differences between groups A, B and $\mathrm{C}$. The P-values of MACE in groups $\mathrm{A}, \mathrm{B}$ and $\mathrm{C}$ were $0.231,2.763$ and 3.142, respectively; whereas the P-values of NACE were 0.157, 0.251 and 0.208, respectively. Significant differences in the frequencies of recurrent hemorrhage were detected among the $\mathrm{A}, \mathrm{B}$ and $\mathrm{C}$ groups $(\mathrm{P}=0.069,0.032$ and 0.040 , respectively). Furthermore, comparing the survival times of patients among the three groups demonstrated that there were significant differences (Table III). The Long-rank and Tarone-Ware methods demonstrated a significant difference between groups A and C, whereas the Breslow method presented a statistical difference between groups A and C,

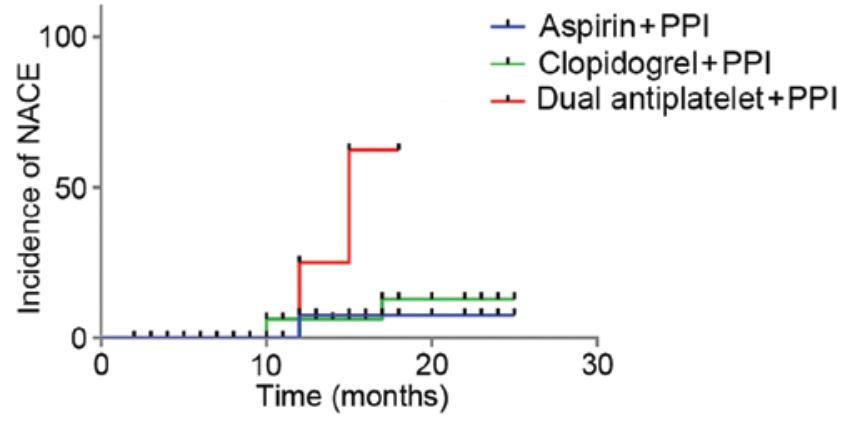

Figure 2. Incidence of net adverse cardiovascular events among the three groups.

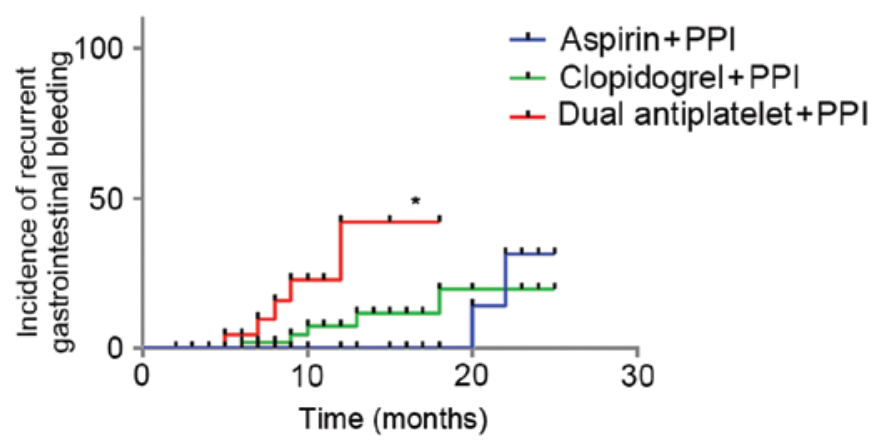

Figure 3. Incidence of recurrent hemorrhage among the three groups. ${ }^{*} \mathrm{P}<0.05$ vs. the aspirin + PPI and clopidogrel + PPI groups.

and groups $\mathrm{B}$ and $\mathrm{C}$. Taken together, these findings indicated that the patients in group A had significantly shorter survival times, as compared with groups B and C. Survival curves are presented in Figs. 1-3. No statistical differences in survival duration after MACE and NACE were detected among three groups, whereas the DAPT group exhibited a significantly reduced survival rate after recurrent hemorrhage. 


\section{Discussion}

It has previously been demonstrated that the administration of any of the commonly recommended basic antiplatelet strategies (5), including aspirin + PPI, clopidogrel + PPI; DAPT + PPI, is associated with a significant reduction of recurrent infarction (9). However, postoperative antiplatelet therapies are associated with digestive tract diseases, resulting in hemorrhage and peptic ulcers, and cardiovascular disease, which may result in MACE (10). As demonstrated in previous large clinical trials (11-16), as compared with aspirin monotherapy, clopidogrel (300 mg followed by $75 \mathrm{mg}$ daily in all except for one of the trials) plus aspirin reduces the risk of composite vascular events [absolute risk reduction, 0.9-6.7\%; relative risk reduction, 8.9-41.9\%]. However, this improvement was associated with an increase in major bleeding events [absolute risk increase, 0.6-2.1\%; relative risk increase, -54.5-37.0\%] over variable periods from 8 days to 12 months. The mechanism of how aspirin may result in digestive diseases, particularly peptic ulcers, can be summarized by the following two points: i) Aspirin directly stimulates the phospholipid layer of gastric mucosa, which damages the hydrophobic protection barrier of the stomach (17), in addition to the increased release of cytotoxic substances (such as leukotrienes), which may also damage the gastric mucosa; ii) aspirin inhibits cyclooxygenase (COX)-1 and COX-2 in gastric mucosa (18). It is well-known that prostaglandin (PG) synthesis requires COX in gastric mucosa, and PGs can increase the blood flow of gastric mucosa and promote the synthesis of the mucus- $\mathrm{HCO}_{3}$ barrier (19). Therefore, when patients are administered aspirin as antiplatelet therapy, $\mathrm{COX}$ is inhibited by aspirin and the gastric mucosa loses the protection of the mucus- $\mathrm{HCO}_{3}$ barrier and peptic ulcers may subsequently develop. Aspirin inhibits platelet aggregation in a dose-dependent manner, and Patrono et al (20) have previously demonstrated that the risk of bleeding increases by 4-6- times when patients are treated with higher doses of aspirin. Conversely, the antiplatelet function of clopidogrel predominantly relies on the irreversible inhibition of the P2Y12 subtype of adenosine (5). Although clopidogrel does not damage the gastric mucosa directly, it may inhibit the release of platelet derived growth factor and vascular endothelial growth factor, resulting in the inhibition of angiogenesis and the healing of peptic ulcers.

Following PCI treatment, various complications may affect the patients' prognosis, particularly bleeding, which may reduce the patients' heart function and lead to MACE. The reason for this can be summarized by the following four points: i) Massive hemorrhage reduces the intravascular volume and increases the heart rate, resulting in an increase of myocardial oxygen consumption and a decrease in myocardial perfusion; ii) in order to treat a massive hemorrhage anticoagulation, antiplatelet and antithrombotic therapy is terminated, which increases the risk of myocardial ischemia and stent thrombosis; and iii) blood transfusion therapy may trigger the release of inflammatory mediators which, in turn, may increase the onset of stent thrombosis (21).

The most salient finding of the present study is that neither monotherapy nor combination therapy was able to induce statistically significant differences in MACE, NACE and recurrent hemorrhage in patients who suffered from gastrointestinal bleeding after PCI surgery and DAPT. These findings may deviate from the results of previous studies; however, the present results can be explained by the combination therapy of PPI. Yasuda et al (22) have previously demonstrated that combination therapy with DAPT and PPI decreases the effect of DAPT; however, the combination therapy was shown to decrease the risk of recurrent hemorrhage. Moreover, previous studies (23-26) have also demonstrated that combination therapy with PPI and clopidogrel reduced the antiplatelet effect of clopidogrel. Therefore, whether combination therapy with clopidogrel and PPI is reasonable remains controversial, and further clinical trials are required.

The present survival analysis results demonstrated that there were significant differences between the aspirin + PPI, clopidogrel + PPI and DAPT + PPI groups. The effect of aspirin + PPI and clopidogrel + PPI combination therapy were demonstrated to be superior to DAPT + PPI combination therapy. No significant differences were detected between the aspirin + PPI and clopidogrel + PPI combination therapy groups. Therefore, we hypothesise that antiplatelet monotherapy is suitable for patients who demonstrate a high risk of gastrointestinal hemorrhage, as compared with DAPT. Furthermore, aspirin is more cost-effective than clopidogrel and patients may prefer it. A 1996 CAPRIE trial (27) demonstrated that upper digestive tract hemorrhage was significantly reduced in patients treated with clopidogrel, as compared with aspirin. These findings are inconsistent with the results of the present study; this may be due to the small number of patients in the aspirin and clopidogrel groups. Cheung et al (28) demonstrated that patients with AMI who are complicated by peptic ulcer hemorrhage should not continue aspirin where viable; whereas patients who are at low risk of peptic ulcers following PCI treatment should regard aspirin as the preferable choice.

The results of the present study indicated that patients who developed gastrointestinal hemorrhage following treatment with DAPT after DES implantation should continue to use antiplatelet monotherapy or DAPT, as no significant differences in the rates of clinical incidents, as determined by MACE, NACE and recurrent hemorrhage, or the results of survival analysis, were detected between the treatment groups. As for the prevention of recurrent bleeding, antiplatelet monotherapy was demonstrated to be superior to DAPT. The treatment of patients who are administered DAPT and experience gastrointestinal hemorrhage following DES implantation must involve an evaluation of the risk of complications, including stent thrombosis, continuous bleeding and recurrent hemorrhage.

\section{Acknowledgements}

The present study was supported by the Department of Health of Guangxi Zhuang autonomous region (grant no. z2008409).

\section{References}

1. Siogkas PK, Papafaklis MI, Sakellarios AI, Stefanou KA Bourantas CV, Athanasiou LM, Bellos CV, Exarchos TP, Naka KK and Michalis LK, et al: Computational assessment of the fractional flow reserve from intravascular ultrasound and coronary angiography data: A pilot study. Conf Proc IEEE Eng Med Biol Soc 2013: 3885-3888, 2013. 
2. Thygesen K, Alpert JS, Jaffe AS, Simoons ML, Chaitman BR, White HD; Joint ESC/ACCF/AHA/WHF Task Force for the Universal Definition of Myocardial Infarction, Katus HA, Lindahl B, Morrow DA, et al: Third universal definition of myocardial infarction. Circulation 126: 2020-2035, 2012.

3. Palmerini T, Benedetto U, Bacchi-Reggiani L, Della Riva D, Biondi-Zoccai G, Feres F, Abizaid A, Hong MK, Kim BK, Jang Y, et al: Mortality in patients treated with extended duration dual antiplatelet therapy after drug-eluting stent implantation: A pairwise and Bayesian network meta-analysis of randomised trials Lancet 385: 2371-2382, 2015.

4. Hallas J, Dall M, Andries A, Andersen BS, Aalykke C, Hansen JM, Andersen M and Lassen AT: Use of single and combined antithrombotic therapy and risk of serious upper gastrointestinal bleeding: Population based case-control study. BMJ 333: 726, 2006

5. Lippi G, Franchini M and Cervellin G: Diagnosis and management of ischemic heart disease. Semin Thromb Hemost 39: 202-213, 2013.

6. Flandre P, Boufassa F, Gerard L, Carre N, Persoz A and Meyer L: The use of auxiliary events to improve the analysis of survival for HIV-infected patients: application to the French Prospective Multicenter Cohort (SEROCO). J Acquir Immune Defic Syndr Hum Retrovirol 12: 174-181, 1996.

7. Sperry SM, Charlton ME and Pagedar NA: Association of sentine lymph node biopsy with survival for head and neck melanoma: survival analysis using the SEER database. JAMA Otolaryngol Head Neck Surg 140: 1101-1109, 2014.

8. Jones MP and Crowley J: A general class of nonparametric tests for survival analysis. Biometrics 45: 157-170, 1989.

9. Matetzky S, Shenkman B, Guetta V, Shechter M, Beinart R, Goldenberg I, Novikov I, Pres H, Savion N, Varon D and Hod H: Clopidogrel resistance is associated with increased risk of recurrent atherothrombotic events in patients with acute myocardial infarction. Circulation 109: 3171-3175, 2004.

10. Hong KS: Dual antiplatelet therapy after noncardioembolic ischemic stroke or transient ischemic attack: Pros and cons. J Clin Neurol 10: 189-196, 2014.

11. Chen ZM, Jiang LX, Chen YP, Xie JX, Pan HC, Peto R, Collins R and Liu LS; COMMIT (ClOpidogrel and Metoprolol in Myocardia Infarction Trial) collaborative group. Addition of clopidogrel to aspirin in 45,852 patients with acute myocardial infarction: Randomised placebo-controlled trial. Lancet 366: 1607-1621, 2005

12. MehtaSR, YusufS, Peters RJ,Bertrand ME, Lewis BS, Natarajan MK, Malmberg K, Rupprecht H, Zhao F, Chrolavicius S, et al: Effects of pretreatment with clopidogrel and aspirin followed by long-term therapy in patients undergoing percutaneous coronary intervention: The PCI-CURE study. Lancet 358: 527-533, 2001.

13. Sabatine MS, Cannon CP, Gibson CM, López-Sendón JL, Montalescot G, Theroux P, Claeys MJ, Cools F, Hill KA, Skene AM, et al: Addition of clopidogrel to aspirin and fibrinolytic therapy for myocardial infarction with ST-segment elevation. N Engl J Med 352: 1179-1189, 2005.

14. Sabatine MS, Cannon CP, Gibson CM, López-Sendón JL, Montalescot G, Theroux P, Lewis BS, Murphy SA, McCabe CH and Braunwald E; Clopidogrel as Adjunctive Reperfusion Therapy (CLARITY)-Thrombolysis in Myocardial Infarction (TIMI) 28 Investigators. Effect of clopidogrel pretreatment before percutaneous coronary intervention in patients with ST-elevation myocardial infarction treated with fibrinolytics: The PCI-CLARITY study. JAMA 294: 1224-1232, 2005
15. Steinhubl SR, Berger PB, Mann JT III, Fry ET, DeLago A, Wilmer C and Topol EJ; CREDO Investigators. Clopidogrel for the Reduction of Events During Observation: Early and sustained dual oral antiplatelet therapy following percutaneous coronary intervention: A randomized controlled trial. JAMA 288: 2411-2420, 2002.

16. Yusuf S, Zhao F, Mehta SR, Chrolavicius S, Tognoni G and Fox KK; Clopidogrel in Unstable Angina to Prevent Recurrent Events Trial Investigators: Effects of clopidogrel in addition to aspirin in patients with acute coronary syndromes without ST-segment elevation. N Engl J Med 345: 494-502, 2001.

17. Mahmud T, Scott DL and Bjarnason I: A unifying hypothesis for the mechanism of NSAID related gastrointestinal toxicity. Ann Rheum Dis 55: 211-213, 1996.

18. Ng SC and Chan FK: NSAID-induced gastrointestinal and cardiovascular injury. Curr Opin Gastroenterol 26: 611-617, 2010.

19. Sakumoto R, Hayashi KG and Takahashi T: Different expression of PGE synthase, PGF receptor, TNF, Fas and oxytocin in the bovine corpus luteum of the estrous cycle and pregnancy. Reprod Biol 14: 115-121, 2014.

20. Patrono C, Bachmann F, Baigent C, Bode C, De Caterina R, Charbonnier B, Fitzgerald D, Hirsh J, Husted S, Kvasnicka J, et al: Expert consensus document on the use of antiplatelet agents. The task force on the use of antiplatelet agents in patients with atherosclerotic cardiovascular disease of the European society of cardiology. Eur Heart J 25: 166-181, 2004.

21. Twomley KM, Rao SV and Becker RC: Proinflammatory, immunomodulating and prothrombotic properties of anemia and red blood cell transfusions. J Thromb Thrombolysis 21: 167-174, 2006.

22. Yasuda H, Yamada M, Sawada S, Endo Y, Inoue K, Asano F, Takeyama Y and Yoshiba M: Upper gastrointestinal bleeding in patients receiving dual antiplatelet therapy after coronary stenting. Intern Med 48: 1725-1730, 2009.

23. Ho PM, Maddox TM, Wang L, Fihn SD, Jesse RL, Peterson ED and Rumsfeld JS: Risk of adverse outcomes associated with concomitant use of clopidogrel and proton pump inhibitors following acute coronary syndrome. JAMA 301: 937-944, 2009.

24. Juurlink DN, Gomes T, Ko DT, Szmitko PE, Austin PC, Tu JV, Henry DA, Kopp A and Mamdani MM: A population-based study of the drug interaction between proton pump inhibitors and clopidogrel. CMAJ 180: 713-718, 2009.

25. Gaglia MA Jr, Torguson R, Hanna N, Gonzalez MA, Collins SD, Syed AI, Ben-Dor I, Maluenda G, Delhaye C, Wakabayashi $\mathrm{K}$, et al: Relation of proton pump inhibitor use after percutaneous coronary intervention with drug-eluting stents to outcomes. Am J Cardiol 105: 833-838, 2010.

26. Gupta E, Bansal D, Sotos J and Olden K: Risk of adverse clinical outcomes with concomitant use of clopidogrel and proton pump inhibitors following percutaneous coronary intervention. Dig Dis Sci 55: 1964-1968, 2010.

27. CAPRIE Steering Committee: A randomised, blinded, trial of clopidogrel versus aspirin in patients at risk of ischaemic events (CAPRIE). CAPRIE Steering Committee. Lancet 348: 1329-1339, 1996.

28. Cheung J, Rajala J, Moroz D, Zhu Q, Stamm M and Sandha GS: Acetylsalicylic acid use in patients with acute myocardial infarction and peptic ulcer bleeding. Can J Gastroenterol 23: 619-623, 2009 